\title{
Thermal Bifunctionality of Bacterial Phenylalanine Aminomutase and Ammonia Lyase Enzymes**
}

\author{
Christopher Chesters, Matthew Wilding, Mark Goodall, and Jason Micklefield*
}

Phenylalanine aminomutases (PAMs) are 4-methylideneimidazol-5-one (MIO)-dependent enzymes ${ }^{[1]}$ that catalyze the isomerization of $(S)$ - $\alpha$-phenylalanine to give $(S)$ - or $(R)-\beta$ phenylalanine, which are precursors in the biosynthesis of various natural products. ${ }^{[2,3]}$ Several related tyrosine aminomutases (TAMs) have also been characterized. ${ }^{[4]}$ Furthermore, the mechanistically related MIO-dependent phenylalanine, tyrosine, and histidine ammonia lyases (PALs, TALs, and HALs) catalyze the elimination of ammonia from $(S)$ - $\alpha$ phenylalanine, $(S)$ - $\alpha$-tyrosine, and $(S)$ - $\alpha$-histidine to give the corresponding trans- $\alpha, \beta$-unsaturated carboxylic acids. ${ }^{[5,6]}$ Two mechanisms have been proposed for the MIO-dependent enzymes (Scheme 1A): ${ }^{[5,7,8]}$ An amino-MIO adduct mechanism, which is supported by crystallographic evidence, including a structure of SgTAM from Streptomyces globispo$r u s,{ }^{[8]}$ and an alternate Friedel-Crafts-type reaction mechanism. ${ }^{[5,7]}$ A recent computational study suggests that both mechanisms could operate, depending on the presence of a specific active-site Glu residue (e.g. Glu484 in the PcPAL of Petroselinum crispum), which is suggested to electrostatically attract the substrate $\alpha$-amino group that favors reaction by the Friedel-Crafts-type mechanism. ${ }^{[9]}$ MIO-dependent enzymes that do not possess a Glu residue at this position are suggested to proceed by the amino-MIO adduct mechanism.

Several studies have sought to discern the structural differences that govern mutase and lyase activity. ${ }^{[4 b, 10]}$ However, attempts to engineer increased lyase activity from existing mutases, based on exchanging conserved sequence motifs using site directed mutagenesis, have so far proved unsuccessful. ${ }^{[4 b, 10]}$ Herein we explore the factors that differentiate MIO-dependent mutase and lyase activity, with a focus on the bacterial enzymes AdmH and EncP, which despite sharing significant sequence similarity $(63 \%$ overall identity and $76 \%$ similarity) are reported to have distinct catalytic functions. Enzyme AdmH from Pantoea agglomerans is a PAM, which provides $(S)$ - $\beta$-phenylalanine required for the biosynthesis of the antibiotic andrimid (Scheme 1B). ${ }^{[2]}$

[*] Dr. C. Chesters, Dr. M. Wilding, M. Goodall, Prof. J. Micklefield School of Chemistry \& Manchester Interdisciplinary Biocentre The University of Manchester

131 Princess Street, Manchester M1 7DN (UK)

E-mail: j.micklefield@manchester.ac.uk

[***] We thank TSB, CoEBio3, and BBSRC for PhD studentships to C.C., M.W., and M.G. We thank Prof. Christopher T. Walsh (Harvard) for AdmH and Prof. Nicholas J. Turner (Manchester) for RgPAL expression plasmids.

9 Supporting information for this article is available on the WWW under http://dx.doi.org/10.1002/anie.201200669.
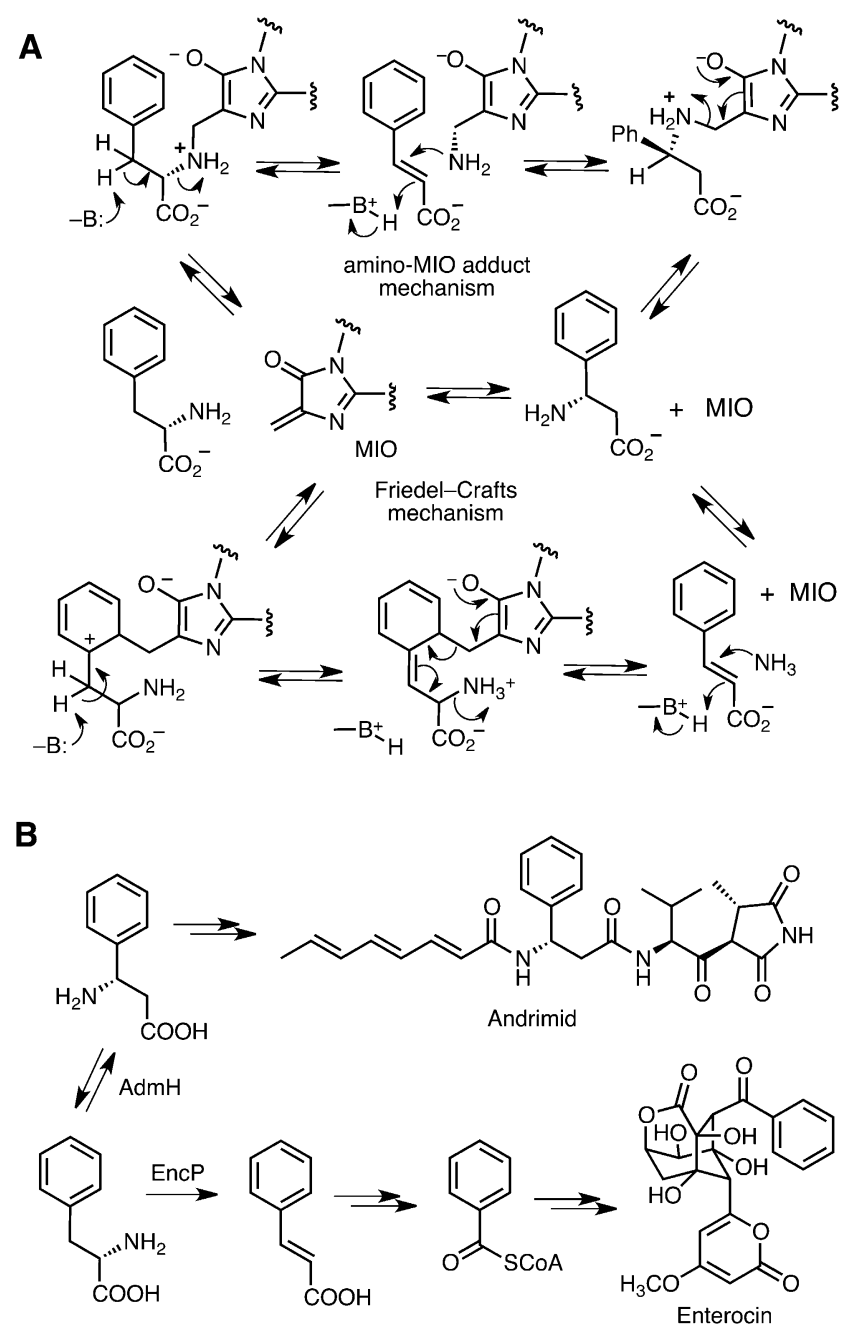

Scheme 1. A) The amino-MIO adduct and Friedel-Crafts-type mechanisms proposed for MIO-dependent lyase and mutase enzymes.

B) The biosynthetic role of $\mathrm{AdmH}$ and EncP. B=base.

Enzyme EncP from the thermotolerant marine bacterium Streptomyces maritimus was shown to function as a PL that produces trans-cinnamate, which is suggested to undergo $\beta$ oxidation to provide the benzoyl-coenzyme A (CoA) starter unit in the biosynthesis of the polyketide enterocin. ${ }^{\left[{ }^{6}\right.}$ Accordingly, enzymes AdmH and EncP were overproduced in E. coli and purified following standard procedures. The kinetic parameters for the turnover of $(S)$ - $\alpha$-phenylalanine to trans-cinnamate ( $\alpha$-lyase activity) catalyzed by EncP were determined spectrophotometrically at $30^{\circ} \mathrm{C}$ (Table 1 ) and are in agreement with published data. ${ }^{[6]}$ Given that $S$. maritimus is 
Table 1: Steady-state kinetic parameters for the phenylalanine ammonia lyase and aminomutase reactions catalyzed by enzymes AdmH and EncP.

\begin{tabular}{|c|c|c|c|c|c|c|c|}
\hline Enzyme & Substrate & $\begin{array}{l}\text { Lyase activity }{ }^{[a]} \\
k_{\text {cat }} \times 10^{-3}\left[\mathrm{~s}^{-1}\right]\end{array}$ & $K_{\mathrm{M}}[\mathrm{mm}]$ & $k_{\text {cat }} / K_{\mathrm{M}}\left[\mathrm{s}^{-1} \mathrm{~mm}^{-1}\right]$ & $\begin{array}{l}\text { Mutase activity }{ }^{[b]} \\
k_{\text {cat }} \times 10^{-3}\left[\mathrm{~s}^{-1}\right]\end{array}$ & $K_{\mathrm{M}}[\mathrm{mm}]$ & $k_{\text {cat }} / K_{\mathrm{M}}\left[\mathrm{s}^{-1} \mathrm{~mm}^{-1}\right]$ \\
\hline \multirow[t]{2}{*}{ AdmH } & $\alpha$-Phe & $3.86( \pm 0.023)$ & $0.027( \pm 0.002)$ & $0.14( \pm 0.008)$ & $87.1( \pm 5.2)$ & $0.032( \pm 0.004)$ & $2.73( \pm 0.17)$ \\
\hline & $\beta$-Phe & $3.18( \pm 0.015)$ & $0.025( \pm 0.001)$ & $0.13( \pm 0.008)$ & $59.0( \pm 2.7)$ & $0.027( \pm 0.003)$ & $2.20( \pm 0.26)$ \\
\hline \multirow[t]{2}{*}{ EncP } & $\alpha$-Phe & $4.77( \pm 0.23)$ & $0.017( \pm 0.002)$ & $0.28( \pm 0.038)$ & $159( \pm 12.1)$ & $0.022( \pm 0.004)$ & $7.19( \pm 0.57)$ \\
\hline & $\beta$-Phe & $6.06( \pm 0.23)$ & $0.025( \pm 0.002)$ & $0.24( \pm 0.022)$ & $220( \pm 15.9)$ & $0.040( \pm 0.004)$ & $5.53( \pm 0.40)$ \\
\hline
\end{tabular}

[a] Deamination of $(S)$ - $\alpha$-phenylalanine $\left(\alpha\right.$-Phe) and $(S)$ - $\beta$-phenylalanine $\left(\beta\right.$-Phe) to give trans-cinnamic acid and ammonia at $30^{\circ} \mathrm{C}$. [b] Isomerization of $\alpha$-Phe $\rightarrow \beta$-Phe and the reverse reaction $\beta$-Phe $\rightarrow \alpha$-Phe at $25^{\circ} \mathrm{C}$. Haldane relationship: $K_{\text {eq }}=\left(k_{\text {cat }} / K_{\mathrm{M}} \alpha \rightarrow \beta\right) /\left(k_{\text {cat }} / K_{\mathrm{M}} \beta \rightarrow \alpha\right) . k_{\text {cat }}=$ turnover number, $K_{\mathrm{M}}=$ Michaelis constant.

known to be thermotolerant, ${ }^{[6]}$ the temperature dependency for the EncP-catalyzed $\alpha$-lyase reaction was explored. This investigation revealed that EncP is a thermophilic enzyme with $\alpha$-lyase activity increasing exponentially from 30 to $64^{\circ} \mathrm{C}$, reaching a maximum activity at $74^{\circ} \mathrm{C}\left(k_{\text {cat }}=0.318 \mathrm{~s}^{-1}\right)$ with approximately 60 -fold higher turnover than observed at $30^{\circ} \mathrm{C}$ (Figure $1 \mathrm{~A}$ ). Enzyme EncP also exhibits significant thermostability, with no loss of activity after incubation at
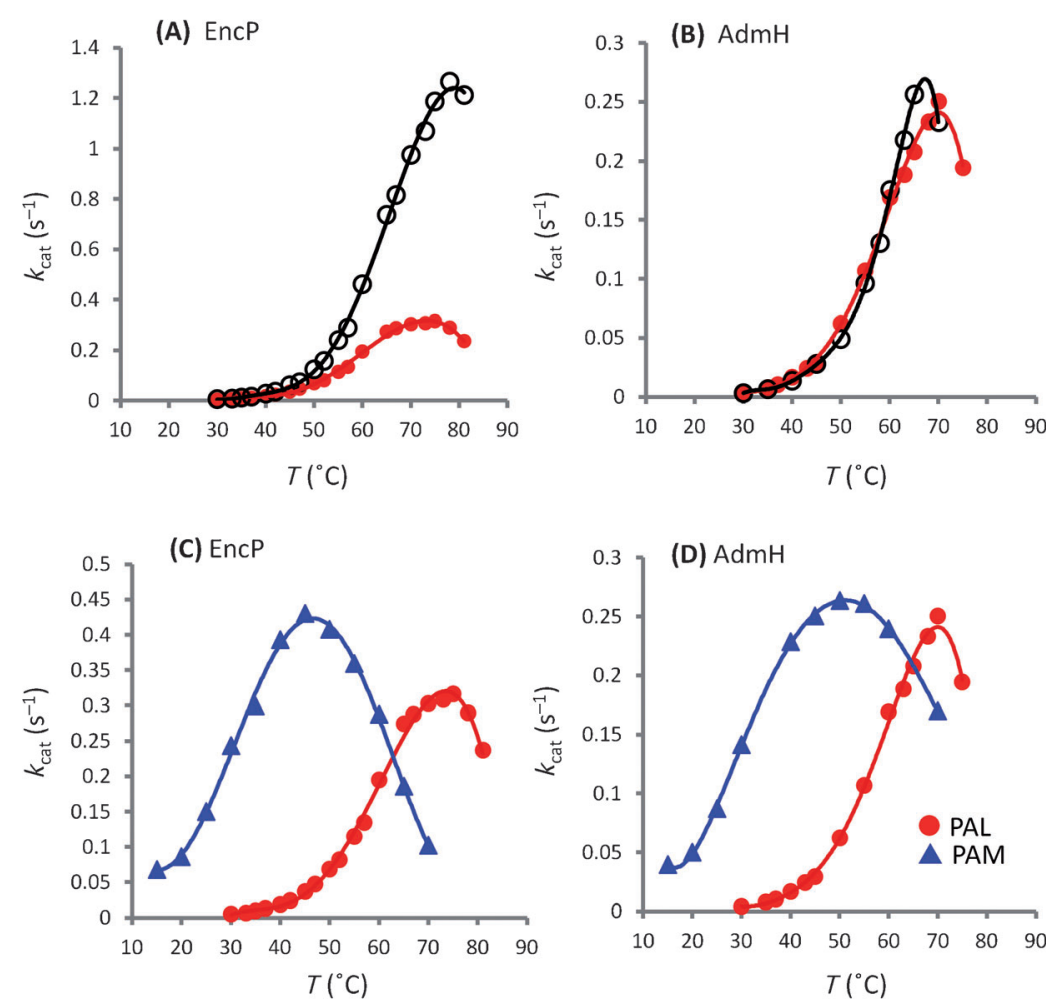

Figure 1. The effect of temperature on $\alpha$-lyase $(\bullet)$ and $\beta$-lyase $(O)$ reactions for $A)$ EncP and $\mathrm{B}) \mathrm{AdmH}$. Comparison of PAL ( $\bullet$ ) and PAM ( $\mathbf{A}$ ) activity of C) EncP and D) AdmH as a function of temperature.

$60^{\circ} \mathrm{C}$ for three hours, whilst $60 \%$ of its original activity was retained after incubation at $65^{\circ} \mathrm{C}$ for two hours. These results are consistent with EncP functioning to yield trans-cinnamate at the high temperatures that might be experienced by the $S$. maritimus host in its natural environment. In agreement with previous studies, ${ }^{[2 a, b]}$ no trans-cinnamate was produced by enzyme AdmH upon incubation with $(S)$ - $\alpha$-phenylalanine at $25^{\circ} \mathrm{C}$, whilst very low PAL activity could be observed at $30^{\circ} \mathrm{C}$ $\left(k_{\text {cat }}\right.$ of $\left.3.86 \times 10^{-3} \mathrm{~s}^{-1}\right)$. However, further increases in temperature provided the unexpected observation that enzyme AdmH has thermophilic PAL activity analogous to EncP (Figure 1B). Moreover, enzyme AdmH also displays significant thermostability, albeit slightly less than that observed for enzyme EncP.

Since $(S)$ - $\beta$-phenylalanine is a known product of $\mathrm{AdmH}$ in the mutase reaction, ${ }^{[2]}$ the AdmH-catalyzed $\beta$-lyase reaction of $(S)$ - $\beta$-phenylalanine to give trans-cinnamate was explored as a function of temperature. The results indicated that the $\beta$-lyase activity of AdmH exhibited a similar temperaturedependence profile to that observed for the $\alpha$ lyase reaction (Figure $1 \mathrm{~B}$ ). Given that enzyme EncP was previously shown to be a PAL, with no reported PAM activity, ${ }^{[6]}$ it was envisaged that this enzyme would not catalyze the $\beta$-lyase reaction with $(S)-\beta$-phenylalanine. Surprisingly, as the temperature is increased, not only does EncP exhibit considerably greater $\beta$-lyase activity than $\alpha$-lyase activity, it also exhibits turnover numbers for the $\beta$-lyase reaction that are approximately 4-5-fold above those observed for AdmH (Figure 1 A,B). Biphasic Arrhenius plots are apparent for both the $\alpha$ - and $\beta$-lyase reactions of $\mathrm{AdmH}$ and $\mathrm{EncP}$, with inflection points at approximately $60-64^{\circ} \mathrm{C}$, which is most likely due to slow thermal denaturation of the enzymes (Figure S1 in the Supporting Information).

In the knowledge that EncP can catalyze both $\alpha$ - and $\beta$-lyase reactions, kinetic parameters for the EncP-catalyzed isomerization of $(S)$ - $\alpha$-phenylalanine to $(S)-\beta$-phenylalanine were determined using an HPLC assay. From this assay it was clear that enzyme EncP is a much more efficient PAM than PAL at $30^{\circ} \mathrm{C}$ and is also a more efficient mutase than $\mathrm{AdmH}$ (Table 1); this finding is surprising given that EncP was reported to function exclusively as a PAL. ${ }^{[6]}$ Moreover, the catalytic rate constants for the PAM activity of enzymes EncP and AdmH were determined as a function of temperature, between 15 and $80^{\circ} \mathrm{C}$ (Figure $1 \mathrm{C}, \mathrm{D}$ ). It is evident that both EncP and $\mathrm{AdmH}$ display remarkably similar temperature-dependency profiles. Notably, both enzymes exhibit predominantly PAM activity at lower temperatures, with very little PAL activity observed. The 
PAM activity for enzymes EncP and AdmH reaches a maximum at approximately $45-50^{\circ} \mathrm{C}$, and at approximately $63-$ $65^{\circ} \mathrm{C}$ both enzymes undergo a transition from PAM to PAL activity, with maximum PAL activity observed at $70-75^{\circ} \mathrm{C}$.

The fact that EncP and AdmH are very similar enzymes, yet fulfill distinct biosynthetic roles, can thus be explained by a novel temperature-dependent switch in activity, which may be governed by differences in the natural environments inhabited by the different bacterial hosts. $S$. maritimus is adapted to survive at high temperatures, which would favor EncP functioning as a PAL, whilst $P$. agglomerans is a mesophile, which is consistent with $\mathrm{AdmH}$ operating as a PAM at lower temperatures. The kinetic parameters for the reverse isomerization of $(S)$ - $\beta$-phenylalanine to $(S)$ - $\alpha$-phenylalanine were also determined (Table 1 ), and by using the Haldane relationship, equilibrium constants were calculated for the mutase reactions catalyzed by enzymes EncP $\left(K_{\text {eq }}=\right.$ $1.31)$ and $\mathrm{AdmH}\left(K_{\mathrm{eq}}=1.24\right)$, which agree closely with the experimental value determined previously with $\operatorname{AdmH}\left(K_{\text {eq }}=\right.$ 1.28 at $\left.25^{\circ} \mathrm{C}\right) .{ }^{[2 \mathrm{a}]}$

We next set out to explore whether the thermal bifunctionality of $\mathrm{AdmH}$ and EncP was also evident in more distantly related MIO-dependent enzymes. For this purpose, a lyase $(R g \mathrm{PaL})$ was chosen from the yeast Rhodotorula graminis. ${ }^{[5,11]} \operatorname{RgPAL}$ was overproduced in E. coli and not surprisingly exhibited $\alpha$-lyase activity, but mutase activity was clearly absent. Despite the absence of mutase activity, RgPAL $\beta$-lyase activity with $(S)$ - $\beta$-phenylalanine was apparent, $k_{\text {cat }}=$ $0.0031( \pm 0.0004) \mathrm{s}^{-1}$, albeit at a rate approximately 800 -fold slower than the $\alpha$-lyase reaction with $(S)$ - $\alpha$-phenylalanine, $k_{\text {cat }}=2.6( \pm 0.09) \mathrm{s}^{-1}$, when determined at the $50^{\circ} \mathrm{C}$ temperature optimum for $\operatorname{RgPAL}$ (Figure S2 in the Supporting Information).

The fact that AdmH, EncP, and RgPAL all catalyze the deamination of $(S)$ - $\beta$-phenylalanine is interesting from a mechanistic perspective. According to the amino-MIO adduct mechanism, both the $\alpha$ - and $\beta$-lyase reactions would be MIO-dependent whilst in the Friedel-Crafts-type mechanism $^{[5]}$ only the $\alpha$-lyase reaction is proposed to involve MIO (Scheme 1 A). To explore these two possibilities further, we sought to determine the effects that deactivating MIO has on the $\beta$-lyase reactions. The MIO cofactor of lyase and mutase enzymes has previously been shown to be sensitive to borohydride reduction. ${ }^{[12]}$ Accordingly, AdmH, EncP, and $R g$ PAL were pretreated with $\mathrm{NaBH}_{4}$, which was found to completely abolish both the $\alpha$ - and $\beta$-lyase activities, thereby establishing stringent MIO dependence. Incubation of enzymes $\mathrm{RgPAL}, \mathrm{AdmH}$, and EncP with saturating levels of either $(S)$ - $\alpha$-phenylalanine or $(S)$ - $\beta$-phenylalanine during pretreatment with $\mathrm{NaBH}_{4}$ provided partial protection of the active site (residual activity of approximately $40 \%$ that of the nontreated enzyme). These results confirmed that the deactivation of the enzymes occurred through a modification to the active site. This finding is interesting given that enzyme RgPAL possesses a Glu residue (Glu301) at the corresponding position to the Glu484 residue of the enzyme PcPAL, which is proposed to sequester the substrate amine and thereby favor the Friedel-Crafts-type mechanism. ${ }^{[9]}$ However, the results presented here indicate that the borohydride treatment abolishes the $\beta$-lyase activity of $R g P A L$; this result is inconsistent with the proposed Friedel-Crafts mechanism, where the $\beta$-lyase reaction should be independent of MIO. Indeed the fact that the enzymes $\mathrm{AdmH}$ and EncP, which are only distantly related to $\mathrm{RgPAL}$, are also dependent on $\mathrm{MIO}$ for $\beta$-lyase activity adds further support to a growing body of evidence in favor of the amino-MIO adduct mechanism for this superfamily of enzymes.

To consider the structural factors that may contribute to the thermal bifunctionality of enzymes AdmH and EncP, homology models (Figure S3 in the Supporting Information) were constructed by using the crystal structure of $\operatorname{SgTAM},{ }^{[8]}$ which exhibits close similarity to AdmH and EncP (38 and $39 \%$, respectively). These homology models showed that each enzyme contains two mobile loops flanking the entrance to the active site: an inner loop containing the proposed catalytic base (Tyr78 in AdmH), as well as an outer loop from an adjacent monomer that spans residues 285-320 in AdmH. Previous molecular dynamic simulations demonstrated that the analogous inner loop of the plant PcPAL undergoes a "breathing" motion that is speculated to facilitate product release. ${ }^{[13]}$ On the basis that movements to the active-site loops would be hindered by increased solvent viscosity, experiments at different solvent viscosities were conducted to probe the nature of the rate-limiting steps for enzymes AdmH and EncP.

Initially, the effects of viscosity $(\eta)$ on the observed kinetic constants for the $\alpha$-lyase reaction of EncP were studied at $55^{\circ} \mathrm{C}$ by varying the concentrations of microviscogens glycerol, sucrose, and PEG300. This study revealed that the $K_{\mathrm{M}}$ value for enzyme EncP was unaffected by solvent viscosity $(\eta)$, thus suggesting that substrate binding is not rate limiting. ${ }^{[14]}$ However, the $k_{\text {cat }}$ value was observed to decrease significantly, in a hyperbolic fashion, with increasing concentrations of each viscogen (Figure S4 in the Supporting Information), a feature that is apparent if Kramers' theory is applicable. A modified version of Kramers' theory postulates that if a conformational change of the enzymes is rate limiting, then $k_{\text {cat }} \propto 1 / \eta^{\beta}$, such that $\ln \left(k_{\text {cat }}\right) \propto-\beta \ln (\eta)$, and the plots of $\ln \left(k_{\text {cat }}\right)$ vs. $\ln (\eta)$ should be linear with a slope of $-\beta .^{[14]}$ It has been shown empirically that the term $\beta$ is a constant that is dependent on the molecular weight of the viscogen, ${ }^{[15]}$ tending toward values of -1 with smaller viscogens, and values approaching 0 with larger viscogens. The plots for the EncP $\alpha$-lyase reaction are clearly linear for all three viscogens, thereby suggesting that the rate-limiting step for the PAL activity of enzyme EncP involves a conformation change in the protein (Figure S6 in the Supporting Information). The $\beta$ values obtained for glycerol, PEG300, and sucrose are $-0.81,-0.52$, and -0.40 , respectively, consistent with the relative molecular weight of each viscogen, thus indicating that the observed reductions in $k_{\text {cat }}$ (Figure S4 in the Supporting Information) are not due to inhibitory effects of the added viscogens. In the case of enzyme AdmH, a linear plot of $\ln \left(k_{\text {cat }}\right)$ vs. $\ln (\eta)$ was also observed for the PAL reaction (Figure S8 in the Supporting Information). Moreover, PAM reactions catalyzed by both EncP and AdmH exhibit a similar linear relationship between $\ln \left(k_{\text {cat }}\right)$ and $\ln (\eta)$ (Figure $S 9$ in the Supporting Information). This linear relationship suggests 
that the rate-limiting steps for the PAL and PAM reactions of EncP and AdmH involve a conformational change of the enzyme.

To establish if the rate-limiting step is solely dependent on conformational changes, kinetic isotope effects on $V_{\max } / K_{\mathrm{M}}$ $\left(V_{\max }=\right.$ maximum velocity) were determined for the PAL reactions of enzymes $\mathrm{AdmH}$ and EncP using [ring,3,3,2$\left.{ }^{2} \mathrm{H}_{8}\right]-(S)-\alpha$-phenylalanine (Figure S11 in the Supporting Information). From this determination $k_{\mathrm{H}} / k_{\mathrm{D}}$ values of $2.0 \pm 0.5$ and $2.5 \pm 0.7$ were observed for the PAL reactions of enzymes AdmH and EncP, respectively.
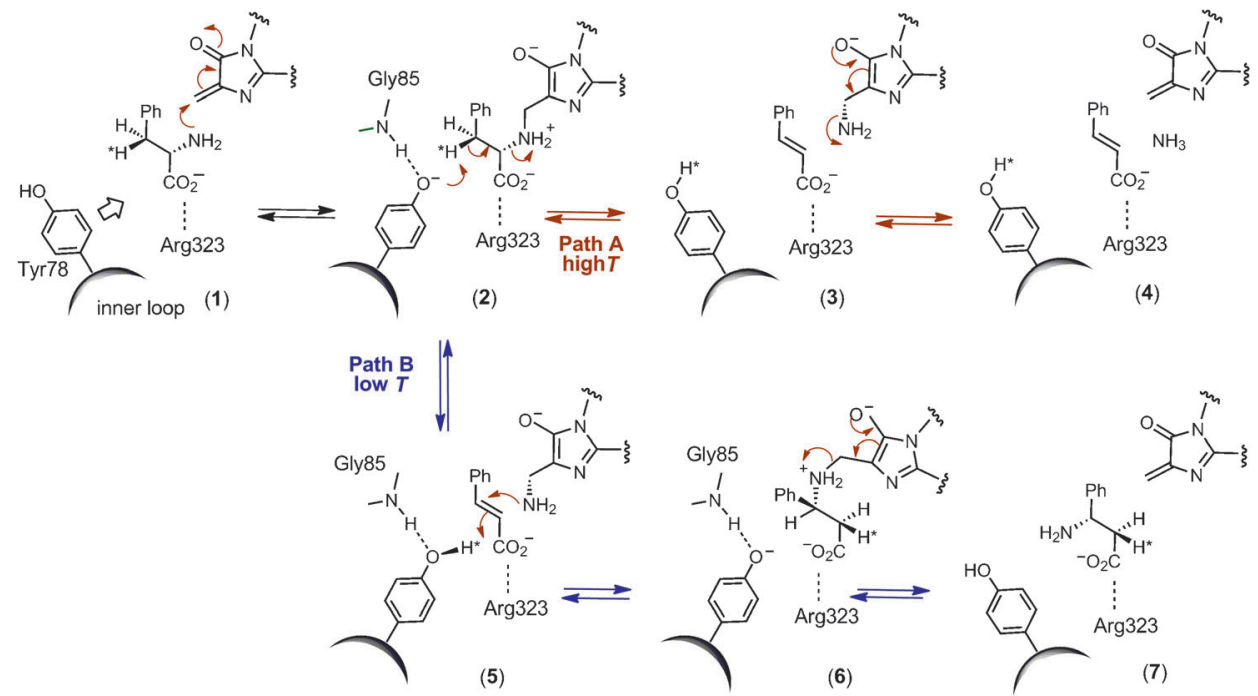

Scheme 2. Proposed mechanism for the PAM and PAL reactions catalyzed by enzymes AdmH and EncP. The stereochemical course of the reaction is based on previous isotopic labeling studies with $\mathrm{AdmH} .{ }^{[2 c]}$

Similar $k_{\mathrm{H}} / k_{\mathrm{D}}$ values of $1.5-2.0$

were reported for related

mutase $^{[3]}$ and lyase ${ }^{[16]}$ enzymes and attributed to the abstraction of the $\mathrm{C} 3$ hydrogen atom being partially rate limiting. ${ }^{[16]}$ The observed kinetic isotope effects, combined with the apparent relationship between kinetics and viscosity $\left(k_{\text {cat }} \propto 1 /\right.$ $\eta^{\beta}$ ), for AdmH- and EncP-catalyzed PAL reactions suggest that both conformational changes and cleavage of the $\mathrm{C} 3-\mathrm{H}$ bond are rate-limiting. One possible explanation for this rate limitation could be that abstraction of the $\mathrm{C} 3$ hydrogen atom during the PAL reaction is coupled with a conformational change of the inner loop, which possesses the catalytic base Tyr78 ( $\mathbf{2} \rightarrow \mathbf{3}$ Scheme 2$)$. Such a conformational change in the mobile inner loop could also facilitate subsequent product release following elimination of ammonia from $\mathrm{MIO}(\mathbf{3} \rightarrow \mathbf{4})$, which is consistent with previous modeling studies with PcPAL. ${ }^{[13]}$ Moreover the fact that the AdmH and EncP PAL reactions are favored at higher temperature is also consistent with this hypothesis (Scheme 2), because at higher temperatures there would be greater thermal energy to overcome the larger energy barrier that is associated with disengaging the inner loop, after abstraction of the C3 hydrogen atom $(\mathbf{2} \rightarrow \mathbf{3})$. At lower temperatures when the PAM reaction predominates, there is presumably insufficient thermal energy to facilitate disengagement of the inner loop, after abstraction of the C3 hydrogen atom. In this case the Tyr 78 hydroxy group would protonate the $\mathrm{C} 2$ carbon atom of cinnamate during conjugate addition of amino-MIO $(\mathbf{5} \rightarrow \mathbf{6})$. Electrostatic repulsion between the resulting Tyr phenolate anion and the carboxylate group of the $\beta$-amino-MIO adduct (6) could then favor disengagement of the inner loop $(\mathbf{6} \rightarrow \mathbf{7})$.

In summary, the enzymes AdmH and EncP display thermal bifunctionality, with mutase activity predominant at lower temperature and lyase activity preferred at higher temperature. This novel temperature-dependent switch in functionality provides a possible explanation of how two very similar enzymes could fulfill distinct catalytic roles in secondary metabolism, which is most likely due to the different environments inhabited by their respective thermo- tolerant and mesophilic bacterial hosts. The effect of temperature on enzyme function has previously been considered with regard to promoting substrate promiscuity ${ }^{[17]}$ and changing enantioselectivity. ${ }^{[18]}$ However to our knowledge, the study presented herein describes the first example of a temperature-dependent switch in enzyme class, which may be related to metabolic function. Moreover we show that AdmH, EncP, and the yeast enzyme RgPAL exhibit MIOdependent $\beta$-lyase activity which is consistent with the amino$\mathrm{MIO}$ adduct mechanism. Our findings that AdmH and EncP display partially rate-limiting conformational changes for both their lyase and mutase activities suggest that there is a high-energy barrier associated with movement of the activesite loops. Moreover, we suggest that the active-site loops play an integral role in differentiating mutase from lyase activity.

Received: January 24, 2012

Published online:

Keywords: aminomutases - ammonia lyases · enzymes . enzyme catalysis $\cdot \beta$-amino acids

[1] a) T. F. Schwede, J. Rétey, G. E. Schulz, Biochemistry 1999, 38, 5355-5361; b) H. A. Cooke, C. V. Christianson, S. D. Bruner, Curr. Opin. Chem. Biol. 2009, 13, 453-461.

[2] a) P. D. Fortin, C. T. Walsh, N. A. Magarvey, Nature 2007, 448, $824-827$; b) N. A. Magarvey, P. D. Fortin, P. M. Thomas, N. L. Kelleher, C. T. Walsh, ACS Chem. Biol. 2008, 3, 542-554; c) N. D. Ratnayake, U. Wanninayake, J. H. Geiger, K. D. Walker, J. Am. Chem. Soc. 2011, 133, 8531-8533.

[3] W. Mutatu, K. L. Klettke, C. Foster, K. D. Walker, Biochemistry 2007, 46, 9785-9794.

[4] a) S. D. Christenson, W. Wu, M. A. Spies, B. Shen, M. D. Toney, Biochemistry 2003, 42, 12708-12718; b) D. Krug, R. Müller, ChemBiochem 2009, 10, 741-750.

[5] L. Poppe, J. Rétey, Angew. Chem. 2005, 117, 3734-3754; Angew. Chem. Int. Ed. 2005, 44, 3668-3688. 
[6] a) L. Xiang, B. S. Moore, J. Biol. Chem. 2002, 277, 32505-32509 b) L. Xiang, B. S. Moore, J. Bacteriol. 2005, 187, 4286-4289; c) L. Xiang, B. S. Moore, J. Bacteriol. 2006, 188, 5331.

[7] J. C. Calabrese, D. B. Jordan, A. Boodhoo, S. Sariaslani, T. Vannelli, Biochemistry 2004, 43, 11403-11416.

[8] a) C. V. Christianson, T. J. Montavon, G. M. Festin, H. A. Cooke, B. Shen, S. D. Bruner, J. Am. Chem. Soc. 2007, 129, $15744-$ 15745 ; b) C. V. Christianson, T. J. Montavon, S. G. Van Lanen, B. Shen, S. D. Bruner, Biochemistry 2007, 46, 7205-7214.

[9] S. Bartsch, U. T. Bornscheuer, Angew. Chem. 2009, 121, 3412 3415; Angew. Chem. Int. Ed. 2009, 48, 3362-3365.

[10] H. A. Cooke, S. D. Bruner, Biopolymers 2010, 93, 802-810.

[11] S. A. Orndorff, N. Costantino, D. Stewart, D. R. Durham, Appl. Environ. Microbiol. 1988, 54, 996.

[12] a) S. D. Christenson, W. Liu, M. D. Toney, B. Shen, J. Am. Chem. Soc. 2003, 125, 6062-6063; b) M. Langer, A. Pauling, J. Rétey,
Angew. Chem. 1995, 107, 1585-1587; Angew. Chem. Int. Ed. Engl. 1995, 34, 1464-1465.

[13] S. Pilbák, A. Tomin, J. Rétey, L. Poppe, FEBS J. 2006, 273, 1004 1019.

[14] a) S. O. Arnett, B. Gerratana, C. A. Townsend, Biochemistry 2007, 46, 9337-9345; b) A. P. Demchenko, O. I. Ru syn, E. A. Saburova, Biochim. Biophys. Acta Protein Struct. Mol. Enzymol. 1989, 998, 196-203.

[15] a) A. Sitnitsky, Physica A 2008, 387, 5483-5497; b) S. Yedgar, C. Tetreau, B. Gavish, D. Lavalette, Biophys. J. 1995, 68, 665-670.

[16] C. B. Klee, K. L. Kirk, L. A. Cohen, Biochem. Biophys. Res. Commun. 1979, 87, 343.

[17] S. Lutz, J. Lichter, L. Liu, J. Am. Chem. Soc. 2007, 129, 87148715.

[18] a) G. Cainelli, P. Galletti, D. Giacomini, Chem. Soc. Rev. 2009, 38, 990-1001; b) T. Sakai, Tetrahedron: Asymmetry 2004, 15, $2749-2756$ 


\section{Angewandte}

Communications

\section{Communications}

Enzyme Catalysis

C. Chesters, M. Wilding, M. Goodall, J. Micklefield*

III-IIII

Thermal Bifunctionality of Bacterial Phenylalanine Aminomutase and Ammonia Lyase Enzymes

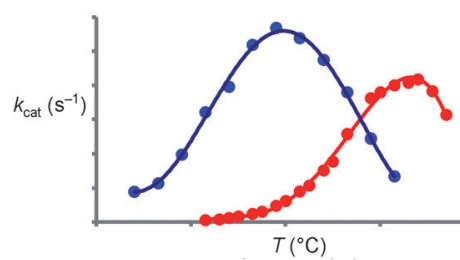

N<smiles>C1CCCC1</smiles>

$\mathrm{COOH}$<smiles>N[C@H](Cc1ccccc1)C(=O)O</smiles>

$\mathrm{Ph} \mathrm{C}_{\mathrm{cooH}}$
Enzymatic thermal switch: The bacterial 4-methylideneimidazol-5-one (MIO)-dependent enzymes $\mathrm{AdmH}$ and EncP are shown to display remarkable thermal bifunctionality: they act as mutases (blue graph) at lower temperatures but with lyase (red graph) activity predominant at higher temperatures. This temperaturedependent switch in enzyme class also explains how these two similar enzymes can fulfill different catalytic functions in secondary metabolisms. 